\title{
SND1 promotes the proliferation of osteosarcoma cells by upregulating COX-2/PGE2 expression via activation of NF- $\mathrm{KB}$
}

\author{
MING ZHOU ${ }^{1,2 *}$, AMAN WANG $^{3 *}$, BAOSHENG YIN $^{4 *}$, DAN WU ${ }^{5}$, SHILIANG HAN ${ }^{2}$, \\ WENPENG ZHANG $^{2}$, JIWEI LIU ${ }^{3}$ and KANG SUN ${ }^{1}$ \\ ${ }^{1}$ Department of Orthopaedics, The Affiliated Hospital of Qingdao University, Qingdao, Shandong 266000; \\ ${ }^{2}$ Department of Orthopaedics, Central Hospital of Zibo, Zibo, Shandong 255036; \\ ${ }^{3}$ Department of Oncology, The First Affiliated Hospital of Dalian Medical University, Dalian, Liaoning 116011; \\ ${ }^{4}$ Department of Orthopaedics, The First Affiliated Hospital of Dalian Medical University, Dalian, Liaoning 116011; \\ ${ }^{5}$ Department of Burns and Plastic Surgery, Central Hospital of Zibo, Zibo, Shandong 255036, P.R. China
}

Received March 8, 2018; Accepted October 1, 2018

DOI: $10.3892 /$ or.2018.6822

\begin{abstract}
Osteosarcoma is the most frequent primary bone tumor. Staphylococcal nuclease domain-containing 1 (SND1) is a multifunctional protein that plays important roles in tumor development and progression. Overexpression of SND1 has been found in several malignancies, however, its expression and function in osteosarcoma is largely unknown. In the present study, we firstly examined the expression of SND1 in 12 pairs of osteosarcoma and healthy bones by immunoblotting and real time-PCR. The results revealed that osteosarcoma tissues expressed significantly high SND1 mRNA and protein expression compared to normal bone tissues. Next, we stably overexpressed SND1 ORF in MG-63 cells and further defined the biological function of SND1 in osteosarcoma by flow cytometry, cell proliferation and in vivo assays. We found that SND1 overexpression significantly promoted cell proliferation and tumor growth in vitro and in vivo. Furthermore, the non-targeted metabolic profiling, ELISA and luciferase reporter assays were performed on stable overexpressing cells and blood samples to elucidate the underlying mechanisms of SND1-mediated oncogenic features. The results revealed that SND1 increased the production of arachidonic acid PGE2.
\end{abstract}

Correspondence to: Professor Jiwei Liu, Department of Oncology, The First Affiliated Hospital of Dalian Medical University, 222 Zhongshan Road, Dalian, Liaoning 116011, P.R. China

E-mail: liujiweidl@126.com

Dr Kang Sun, Department of Orthopaedics, The Affiliated Hospital of Qingdao University, 16 Jiangsu Road, Qingdao, Shandong 266000, P.R. China

E-mail: sunkang_qy@aliyun.com

*Contributed equally

Key words: SND1, COX-2, PGE2, osteosarcoma, NF-кB, proliferation
The serum PGE2 expression level had a significant positive association with the SND1 mRNA expression level in osteosarcoma tissues. The SND1 overexpression-stimulated cell proliferation was enhanced by exogenous addition of PGE2. Additionally, we found that SND1 upregulated PGE2 expression through the $\mathrm{NF}-\kappa \mathrm{B} /$ cyclooxygenase-2 (COX-2) pathway. In summary, our findings revealed the mechanisms of SND1 involvement in osteosarcoma tumor development, and support the targeting of SND1 as a new anti-tumor strategy for patients with osteosarcoma. In addition, SND1 may act as a potential biomarker of the therapeutic strategies utilizing COX-2 inhibitors.

\section{Introduction}

Osteosarcoma is the most common primary malignant tumor of the bones, which usually occurs in the rapidly growing backbone epiphysis (1). Despite improvements in the efficacy of surgery combined with radiotherapy and/or chemotherapy, the 5-year survival rate of metastatic osteosarcoma patients is still less than $20 \%$. Most patients with osteosarcoma succumb from lung metastases. At initial diagnosis, approximately $20 \%$ of patients present with lung metastases whereas $40 \%$ of patients develop metastases at a later stage (2). There are currently no approved targeted therapies for osteosarcoma. Therefore, identifying the molecular mechanisms underlying osteosarcoma growth and metastasis is important to facilitate the development of effective therapeutic strategies of osteosarcoma.

COX-2 is an important rate-limiting enzyme that metabolizes arachidonic acid (AA) to endogenous prostaglandin (PG) (3). Overexpression of COX-2 mainly enhances the activation of the AA/PG pathway and accelerates the production of PGE2, thus promoting tumorigenesis (4). The COX-2/AA/PGE2 pathway is involved in inflammatory response, carcinogenesis, angiogenesis, invasion and metastasis of several tumors (5-7). Exogenous PGE2 promotes the growth of osteosarcoma and represses the effects of meloxicam on cell viability (8). Recent 
studies have shown that the percentage of osteosarcoma with COX-2 positivity ranges from 67 to $92 \%$, and it is greater in metastatic lesions than that of the primary site $(9,10)$. The inhibition of COX-2/PGE2 expression has become one of the promising targets for the treatment of osteosarcoma.

SND1 is a multifunctional protein involved in multiple cellular processes, such as cell differentiation, cell proliferation, adipogenesis and lipid droplets in cellular stress responses. Initially, SND1 was identified as a transcriptional co-activator of Epstein-Barr virus nuclear antigen 2 (EBNA2) and subsequently found to interact with other transcription factors, such as c-Myb, Pim-1, signal transducer and activator of transcription 5 (STAT5), and STAT6 (11). In addition, SND1 is a component of the RNA-induced silencing complex (RISC) that regulates RNAi-mediated gene silencing (12). Recent studies have shown that SND1 is highly expressed in multiple types of cancer, including liver, colon, breast and prostate cancer and glioma (13). To date, the role of SND1 in the carcinogenic process is not well understood.

The NF- $\kappa$ B pathway is one of the most important pathways involved in inflammation and carcinogenesis (14). Inhibition of the NF- $\kappa \mathrm{B}$ pathway represses the growth and increases the sensitivity of osteosarcoma to chemotherapies in vitro and in vivo (15). A recent study has revealed that SND1 promoted the development of hepatocellular cancer by activating $\mathrm{NF}-\kappa \mathrm{B}$ pathways (16). The activation of $N F-\kappa B$ is involved in the transcription of $P T G S 2$, which is the encoding gene of COX-2 protein. In our preliminary experiments, we detected the protein level of SND1 and COX-2 in 12 fresh osteosarcoma tissues by western blotting and found that the expression of these two proteins was highly consistent. Therefore, we hypothesized that SND1 may promote COX-2 expression via the $\mathrm{NF}-\kappa \mathrm{B}$ pathway and participate in the development of osteosarcoma.

The present study revealed that the SND1 protein was upregulated in osteosarcoma tissues compared to paired normal bone tissues. Moreover, SND1 promoted osteosarcoma growth in vitro and in vivo by upregulating $\mathrm{NF}-\kappa \mathrm{B}$ activity to subsequently promote the expression of COX-2/PGE2. Collectively, these functional and biochemical studies indicated that SND1 exhibited oncogenic activities in osteosarcoma, and supported the targeting of SND1 as a new antitumor strategy for patients with osteosarcoma.

\section{Materials and methods}

Patients and tissue/blood samples. Fresh osteosarcoma tissues, normal bone tissues and paired blood samples were obtained from 12 patients with osteosarcoma who underwent surgical resection from January 2014 to January 2016 at the Department of Osteology of the First Affiliated Hospital of Dalian Medical University. Information on patient demographics (sex and age) and clinicopathological features (histology type and Ennecking stage) were obtained from clinical and pathological records (Table I). There were 7 male and 5 female patients with ages ranging from 12 to 33 years. All fresh samples were collected from the patients at the time of surgical resection and confirmed by hematoxylin and eosin staining in frozen sections with histopathological analysis at the Department of Pathology of the First Affiliated Hospital of Dalian Medical University. The distal tissue of the resected tissue was collected and confirmed to be normal bone by frozen section. Twenty-three blood samples from healthy volunteers were obtained from the Physical Examination Center of the First Affiliated Hospital of Dalian Medical University. Blood samples were centrifuged at $4^{\circ} \mathrm{C}$ and $1,080 \mathrm{xg}$ for $10 \mathrm{~min}$ to obtain serum, and samples were stored at $-80^{\circ} \mathrm{C}$ until their use. Additionally, all of these surgical patients signed informed consents and agreed to donate their tissues and blood for research. Ethical approval for the project was obtained from the Research Ethics Committee of the First Affiliated Hospital of Dalian Medical University.

Cell culture. The human osteosarcoma cell lines, HOS, Saos-2 and MG-63 were purchased from the Cell Bank of the Committee on Type Culture Collection of the Chinese Academy of Sciences (Shanghai, China). HOS and MG-63 cells were cultured in MEM medium supplemented with $10 \%$ fetal bovine serum (FBS; both from GIBCO; Thermo Fisher Scientific, Inc., Waltham, MA, USA). Saos-2 cells were maintained in McCoy's 5A medium (Sigma-Aldrich; Merck KGaA, Darmstadt, Germany) supplemented with $10 \%$ FBS. All the cells were cultured in cell culture flasks or Petri dishes in a humidified incubator at $37^{\circ} \mathrm{C}$ in an atmosphere of $5 \% \mathrm{CO}_{2}$.

Plasmids and transfection. The human SND1 ORF was obtained from Shanghai GenePharma Co., Ltd. (Shanghai, China) and subcloned into the pLOC-Lentivirus vector. All transfection reactions were performed using PEI (Invitrogen; Thermo Fisher Scientific, Inc.) in accordance with the manufacturer's instructions. Stable transfectants were selected with $10 \mu \mathrm{g} / \mathrm{ml} \mathrm{BSH}$ (Invitrogen; Thermo Fisher Scientific, Inc.).

siRNA and transfection. siRNA for P65 (5'-GATCCGAAG ACAGCCTTTACTGAAATTCAAGAGATTTCAGTAAAG GCTGTCTTTTTTTTG-3') and a negative control were purchased from Shanghai GenePharma Co., Ltd. (Shanghai, China). According to the manufacturer's protocol, cells were transfected using Lipofectamine 2000 (Invitrogen; Thermo Fisher Scientific, Inc.). After transfection, the cells were cultured for 48 or $72 \mathrm{~h}$ before use in further ELISA and immunoblotting experiments.

RNA isolation and quantitative real-time PCR. Total RNA was isolated from tumor cells using TRIzol reagent (Invitrogen; Thermo Fisher Scientific, Inc.). Reverse transcription PCR was performed using the Revert Aid First Strand cDNA synthesis kit (Fermentas; Thermo Fisher Scientific, Inc.) according to the manufacturer's protocol. Quantitative real-time PCR was performed using StepOnePlus and the DNA double-strand-specific reagent SYBR-Green I for detection (Roche Applied Science, Penzberg, Germany). Fold changes were calculated using the $\mathrm{Cq}$ method (17). The results were normalized to GAPDH levels. The primer sequences were as follows: SND1 forward, GTGATCAGATACCGGCAGGATG and reverse, TCTTAATAGCTCTGGCCTCTGCAG; GAPDH forward, CACCATTGGCAATGAGCGGTTC and reverse, AGGTCTTTGCGGATGTCCACGT.

Flow cytometric analysis. Cells pellets were washed with phosphate buffered saline and fixed/permeabilized with 
Table I. SND1 expression in patients suffering with osteosarcoma.

\begin{tabular}{|c|c|c|c|c|}
\hline \multirow[b]{2}{*}{ Characteristics } & \multirow[b]{2}{*}{$\mathrm{N}$} & \multicolumn{2}{|c|}{ SND1 } & \multirow{2}{*}{$\begin{array}{c}\text { High SND1 } \\
\text { expression rate }(\%)\end{array}$} \\
\hline & & High & Low & \\
\hline \multicolumn{5}{|l|}{ Ages (years) } \\
\hline$\leq 20$ & 8 & 5 & 3 & 62.5 \\
\hline$>20$ & 4 & 1 & 3 & 25 \\
\hline \multicolumn{5}{|l|}{ Sex } \\
\hline Male & 7 & 4 & 3 & 57 \\
\hline Female & 5 & 2 & 3 & 40 \\
\hline \multicolumn{5}{|l|}{ Histology type } \\
\hline Osteoblastoma & 5 & 4 & 1 & 80 \\
\hline Chondroblastic & 3 & 1 & 2 & 33.3 \\
\hline Fibroblastic & 4 & 1 & 3 & 25 \\
\hline \multicolumn{5}{|l|}{ Ennecking stage } \\
\hline IA & 2 & 1 & 1 & 50 \\
\hline IIA & 3 & 1 & 2 & 33.3 \\
\hline IB & 3 & 1 & 2 & 33.3 \\
\hline IIB & 4 & 3 & 1 & 75 \\
\hline
\end{tabular}

SND1, staphylococcal nuclease domain containing 1.

$50 \%$ ice-cold ethanol. Pellets were washed and resuspended in $50 \mu \mathrm{g} / \mathrm{ml}$ ribonuclease A and $62.5 \mu \mathrm{g} / \mathrm{ml}$ propidium iodide. Samples were analyzed on the BD FACSCalibur (BD Biosciences, San Jose, CA, USA). The percentages of cells in various phases of the cell cycle were quantified using the ModFit LT Version 3.0 program (Verity Software House, Inc., Topsham, ME, USA). The error bars were derived from the SD of multiple experiments.

Luciferase reporter assay. X-tremeGene9 (Sigma-Aldrich; Merck KGaA) was used for the cell transfection. The firefly luciferase reporter gene construct, $3 \kappa \mathrm{B}$-Luc, containing three tandem NF- $\kappa \mathrm{B}$-binding sites upstream of the luciferase gene, as well as a Renilla luciferase expression construct $(0.5 \mu \mathrm{g})$ and pRL-SV40 Renilla luciferase construct (10 ng, for normalization) (pSV40-h-gal; Promega Corp., Madison, WI, USA) were used for co-transfection. Cell extracts were prepared $48 \mathrm{~h}$ after transfection. The luciferase activity was measured using the Dual-Luciferase Reporter Assay System (Promega Corp.) according to the manufacturer's protocol (18). The h-galactosidase activity was determined using the Galacto-Light Plus kit (Tropix, Inc., Bedford, MA, USA).

Cell proliferation assay. Firstly, equal numbers of cells were plated in 12-well plates in triplicate. Beginning on day 3 , the cells were fixed with $10 \%$ methanol and stained with $0.1 \%$ crystal violet (dissolved in 10\% methanol) every day. After staining, wells were washed three times with phosphate-buffered saline (PBS) and destained with acetic acid, and the absorbance of the crystal violet solution was measured at $590 \mathrm{~nm}$.
ELISA. Culture supernatants were harvested, centrifuged to remove cellular debris, and stored at $-80^{\circ} \mathrm{C}$. Culture supernatants and serum levels of PGE2 were quantified using a commercial ELISA kit (cat. no. ab133021; Abcam, Cambridge, UK), according to the manufacturer's instructions.

\section{LC-MS based metabolomics analyses}

Cell sample preparation. Cell culture plates were washed with PBS and snap-frozen in liquid nitrogen and stored at $-80^{\circ} \mathrm{C}$. To the culture plate, $1 \mathrm{ml}$ of $-20^{\circ} \mathrm{C}$ pre-cooled $80 \%$ methanol [with internal standard mixture of carnitine $\mathrm{C} 2: 0-\mathrm{d} 3$, carnitine C10:0-d3, carnitine C16:0-d3, LPC 19:0, free fatty acid (FFA) 16:0-d3, FFA 18:0-d3, chenodeoxycholic acid-d4, cholic acid-d4, leu-d3, phe-d5 and tryptophan-d5] was added, and cells were gently scraped off the bottom of the plate into a 5 -ml EP tube using a cell scraper. After thorough vortexing and centrifugation $\left(13,000 \mathrm{x} \mathrm{g}\right.$ for $10 \mathrm{~min}$ at $\left.4^{\circ} \mathrm{C}\right)$, the supernatant was pipetted for drying into a CentriVap Centrifugal Vacuum Concentrator (Labconco Corp., Kansas City, MO, USA). The dried residues were stored at $-80^{\circ} \mathrm{C}$ until analysis.

LC-MS-based metabolic profiling. For LC-MS-based metabolic profiling, an ultra-high-performance liquid chromatograph (UPLC) (Waters Corp., Milford, MA, USA) was coupled to a triple TOF ${ }^{\mathrm{TM}} 5600$ plus (Applied Biosystems, Foster City, CA, USA) mass spectrometer equipped with an electrospray source. The dried sample powder was dissolved in $80 \mu \mathrm{l}$ of acetonitrile/water $(1: 4, \mathrm{v} / \mathrm{v})$. After centrifugation at $13,000 \mathrm{x} \mathrm{g}$ for $10 \mathrm{~min}$ at $4^{\circ} \mathrm{C}, 5 \mu \mathrm{l}$ of supernatant was injected. During LC separation, the column temperature was set at $50^{\circ} \mathrm{C}$, with an elution rate of $0.35 \mathrm{ml} / \mathrm{min}$. For the positive mode, a BEH C8 (100x2.1 mm x1.7 $\mu \mathrm{m}$; Waters Corp.) column was used. The gradient started with $10 \% \mathrm{~B}(0.1 \%$ formic acid in acetonitrile) for $1 \mathrm{~min}$, linearly increased to $40 \% \mathrm{~B}$ within $4 \mathrm{~min}$, then increased to $100 \% \mathrm{~B}$ at $17 \mathrm{~min}$ and maintained for 5 min. The elute phase was rapidly changed to $90 \%$ A $(0.1 \%$ formic acid in water) (within $0.1 \mathrm{~min}$ ), and the total run time for each injection was $25 \mathrm{~min}$, including a post-equilibration of $2.9 \mathrm{~min}$. For the negative mode, an HSS T3 $(100 \times 2.1 \mathrm{~mm}$ $\mathrm{x} 1.8 \mu \mathrm{m}$; Waters Corp.) column was used. The gradient started with $0 \% \mathrm{D}\left[6.5 \mathrm{mM} \mathrm{NH}_{4} \mathrm{HCO}_{3}\right.$ in methanol/water $\left.(95 / 5, \mathrm{v} / \mathrm{v})\right]$ for $1 \mathrm{~min}$, linearly increased to $40 \% \mathrm{D}$ within $2 \mathrm{~min}$, then increased to $100 \% \mathrm{D}$ at $16 \mathrm{~min}$ and maintained for $6 \mathrm{~min}$. The elute phase was rapidly changed to $100 \%$ Av $(6.5 \mathrm{mM}$ $\mathrm{NH}_{4} \mathrm{HCO}_{3}$ in water) (within $0.1 \mathrm{~min}$ ), and the total run time for each injection was $25 \mathrm{~min}$, including a post-equilibration of $2.9 \mathrm{~min}$.

For data acquisition, the $\mathrm{m} / \mathrm{z}$ values were scanned from 50-1200 for both ESI+ and ESI- modes. The ion source parameters were as follows: Source temperature set of $500^{\circ} \mathrm{C}$; Gas 1 and Gas 2 values were both $0.28 \mathrm{MPa}$; curtain gas was $0.24 \mathrm{MPa}$; and the floating ion spray voltages were $5,500 \mathrm{~V}$ and $-4,500 \mathrm{~V}$ for positive and negative ion modes, respectively. IDA-based auto-MS2 was performed for the top 20 ions in MS response with an $\mathrm{m} / \mathrm{z}$ scanning range of 50-1,200 Dalton, collision energy of $( \pm) 30 \mathrm{~V}$ and collision energy spread of 10 V. (19).

$T N F-\alpha$ treatment. To activate the NF- $\kappa \mathrm{B}$ signaling pathway, exogenous TNF- $\alpha$ was applied to the related experiments. 
Following the transient transfection of a firefly luciferase reporter gene for $48 \mathrm{~h}, 10 \mathrm{ng} / \mathrm{ml} \mathrm{TNF}-\alpha$ was added to the culture medium for $12 \mathrm{~h}$. Then, cell extracts were prepared, and the luciferase activity was assessed. In addition, $10 \mathrm{ng} / \mathrm{ml} \mathrm{TNF}-\alpha$ was also added to the culture medium of control or SND1 overexpressing MG-63 cells with or without p65-siRNA treatment for $24 \mathrm{~h}$. Subsequently, the cell extracts were prepared for the further ELISA and immunoblotting experiments.

Western blot analysis. Western blot analyses were performed with standard methods. Briefly, cells were lysed in radioimmunoprecipitation assay (RIPA) buffer containing protease inhibitors and phosphatase inhibitors (Sigma-Aldrich; Merck KGaA, Darmstadt, Germany). Protein concentrations were measured using the BCA assay. Equal amounts of protein $(10 \mu \mathrm{g})$ were fractionated by SDS-PAGE (10 or 12\%) and then transferred to nitrocellulose membranes (Bio-Rad Laboratories, Inc., Hercules, CA, USA). The membranes were blocked in a buffer (TBS; $50 \mathrm{mM}$ Tris- $\mathrm{HCl}$ and $150 \mathrm{mM}$ $\mathrm{NaCl}, \mathrm{pH} 7.4$ ) containing $5 \%$ bovine serum albumin (BSA) and $0.1 \%$ Tween-20 followed by incubation with the following primary antibodies SND1 (dilution 1:1,000; cat. no. sc-271590; Santa Cruz Biotechnology, Inc., Santa Cruz, CA, USA), COX-2 (dilution 1:1,000; cat. no. sc-271590), p65 (dilution 1:1,000; cat. no. 10745-1-AP) and PCNA (dilution 1:1,000, cat. no. 10205-1-AP; all from ProteinTech Group, Inc., Wuhan, China), cyclin A (dilution 1:500; cat. no. sc-136253; Santa Cruz Biotechnology, Inc.), cyclin B1 (dilution 1:1,000; cat. no. 55004-1-AP), cyclin D1 (dilution 1:1,000, cat. no. 60186-1-Ig) and cyclin E (dilution 1:1,000, cat. no. 11554-1-AP; all from ProteinTech Group, Inc.), $\beta$-actin (dilution 1:1,000, cat. no. sc-8432; Santa Cruz Biotechnology, Inc.) and GAPDH (dilution 1:3,000, cat. no. ab8245; Abcam). The immunoreactive proteins were visualized using the ECL western blot analysis system (Bio-Rad Laboratories, Inc.) and densitometric analysis was performed using the Image Pro-Plus software (Media Cybernetics, Inc., Rockville, MD, USA).

In vivo assays for tumor growth. Pathogen-free female athymic nude mice (4 weeks old; 18-22 g) were purchased from the Beijing Vital River Laboratory Animal Technology Co., Ltd. (Beijing, China). All the mice were housed in specific pathogen-free (SPF) environments at the Institute of Genome Engineered Animal Models for Human Disease of Dalian Medical University. All animal experiments were performed in accordance with a protocol approved by Dalian Medical University Research Ethics Committee. MG-63-control or MG-63-SND1 cells were implanted into the right or left dorsal flank of 4-week-old female nude mice. Based on the mean tumor volume at each time-point, growth curves were plotted for each experimental group. The tumor dimensions were measured every 3 days using a digital caliper. The tumor volume $\left(\mathrm{mm}^{3}\right)$ was calculated as follows: $\mathrm{V}=\mathrm{ab}^{2} / 2$ where a and $b$ were the largest and smallest tumor diameters measured at necropsy, respectively. After 22 days, the mices were sacrificed by $\mathrm{CO}_{2}$ asphyxiation and the tumor tissues were harvested for use in further ELISA and immunoblotting experiments.

According to the animal ethical guidelines and our preliminary experimental results, we set the maximum tumor weight to $10 \%$ of the mouse weight (equivalent to a maximum tumor diameter of $20 \mathrm{~mm}$ ) as the experimental endpoint. In fact, the maximum tumor burden observed in our study was $7.6 \%$ and no mouse in the study presented with multiple tumors. The non-retrospective ethical approval obtained for the animal experiments was obtained from the Research Ethics Committee of Dalian Medical University.

Statistical analysis. All data represented the mean \pm standard deviation of at least three independent experiments. Student's t-test was used to assess the differences between two groups. ANOVA-post-hoc pairwise comparison analysis was used to compare the means from three groups. Pearson's correlation analysis was used to assess the correlation between two groups. $\mathrm{P}<0.05$ was considered to indicate a statistically significant difference. All of the relative protein expression was normalized by ImageJ (version no.: 1.8.0_112; https://imagej.nih. gov/ij/). Statistical analysis was performed using the SPSS 18.0 software package (SPSS, Inc., Chicago, IL, USA).

\section{Results}

SND1 expression in fresh osteosarcoma tissues and adjacent normal tissues. To determine if SND1 is dysregulated in osteosarcoma tissues, immunoblotting and real time-PCR analyses were performed using human osteosarcoma tissues and paired normal bone tissues. As revealed in Fig. 1A, 75\% of osteosarcoma samples exhibited high SND1 expression compared to normal bone tissues. Osteosarcoma tissues expressed significantly high SND1 mRNA (Fig. 1B) and protein expression (Fig. 1C), which was increased by 1.74 - and 3.07 -fold, respectively, compared to normal bone tissues.

Osteosarcoma is classified into chondroblastic, osteoblastoma, telangiectatic and fbroblastic types according to the histological difference. Immunoblotting data revealed that SND1 was highly expressed in $80 \%$ of osteoblastoma cases. However, SND1 expression was present in only 33.3 and $25 \%$ in chondroblastic and fbroblastic types cases, respectively. In the II B phase of Ennecking staging, positive SND1 cases accounted for $75 \%$, which was far higher than other stages (Table I). Thus, these results revealed that high SND1 expression may be related to the histological classification and the Ennecking stage of osteosarcoma.

SND1 promotes the proliferation of osteosarcoma cells. To investigate the role of SND1 in osteosarcoma cells, the protein expression of SND1 was determined in 3 osteosarcoma cell lines, namely MG-63, Saos-2 and HOS. SND1 expression was relatively lower in MG-63 cells compared to Saos-2 and HOS cells (Fig. 2A). To further define the biological function of SND1 in osteosarcoma, SND1 was stably overexpressed in MG-63 cells, which were transfected with SND1-pLOC-Lentivirus plasmids and selected with $10 \mu \mathrm{g} / \mathrm{ml} \mathrm{BSH}$. Western blot analysis was used to verify the transfection efficiency. As shown in Fig. 2B, the SND1 protein expression in MG63-SND1 cells was significantly higher compared to the mock and control cells which were stably transfected with a pLOC-lentiviral vector containing an RFP open reading frame. Next, cell populations were examined by flow cytometry. SND1 overexpression increased the 


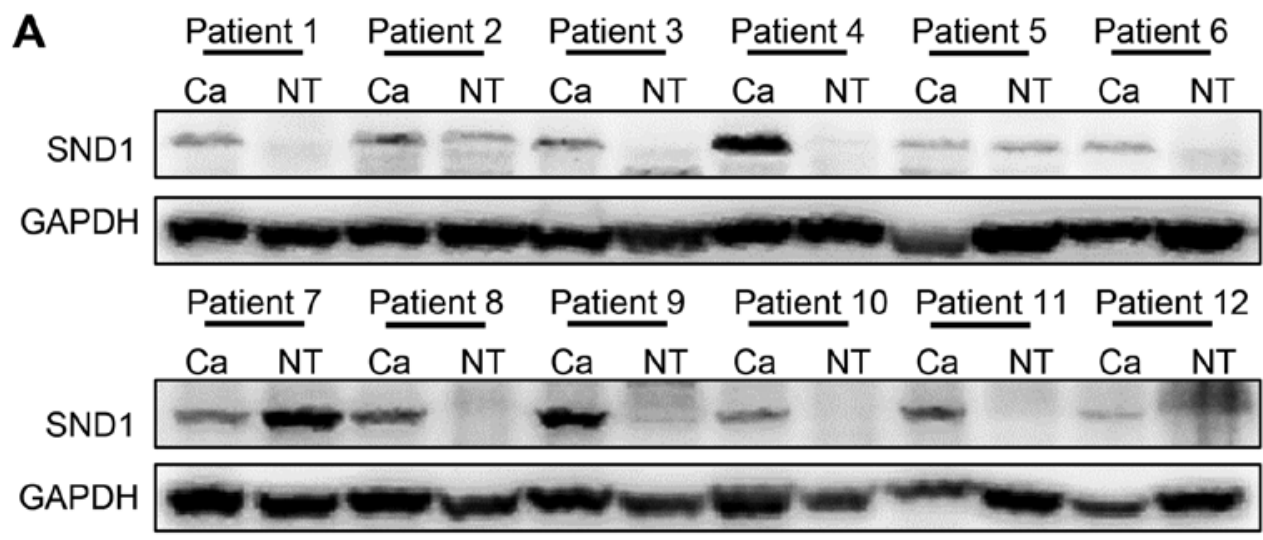

B

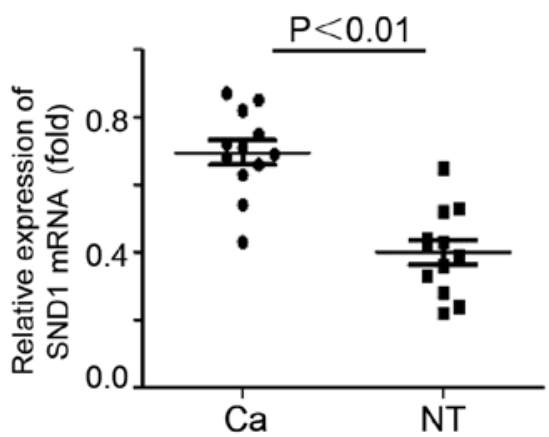

C

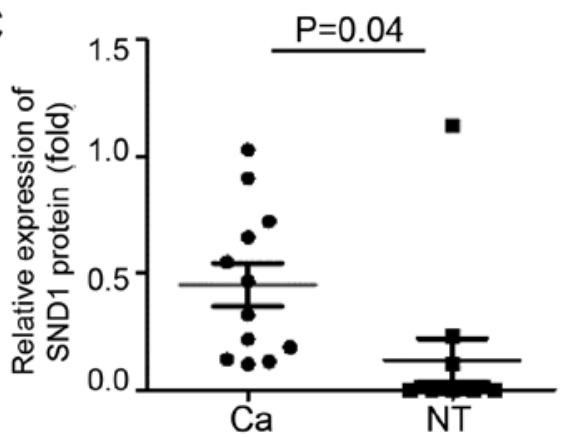

Figure 1. SND1 expression in fresh osteosarcoma tissues and paired normal bone tissues. (A and B) Western blot analysis of SND1 expression in 12 pairs of osteosarcoma fresh-frozen tissues and normal bone tissues. (C) qRT-PCR analysis of SND1 mRNA expression in 12 pairs of osteosarcoma fresh-frozen tissues and the paired normal bone tissues. All of the relative protein expression was normalized by ImageJ (version no.: 1.8.0_112, https://imagej.nih.gov/ij/). Statistical significance was determined by a two-tailed, paired Student's t-test in B and C. SND1, staphylococcal nuclease domain-containing 1.

G1/S transition rate, resulting in a significant decrease in the proportion of G1 phase cells and an increase in the proportion of S phase cells (Fig. 2C). Additionally, SND1 overexpression significantly stimulated proliferation compared to the control cells (Fig. 2D). Cyclin proteins in SND1-overexpressing and control cells were also detected. As shown in Fig. 2E, overexpression of SND1 led to a significant increase of cyclin D1 and cyclin E, while cyclin A and cyclin B1 were not altered. Collectively, these findings indicated that SND1 promoted the proliferation of osteosarcoma cells.

SND1 promotes PGE2 synthesis and release. To elucidate the underlying mechanisms of SND1-mediated oncogenic features, we applied untargeted metabolite profiling in control and SND1-overexpressing cells using LC-MS. Arachidonic acid and other 20-C polyunsaturated fatty acids were upregulated in SND1-overexpressing cells compared to control cells (Fig. 3A). In addition, the expression level of PGE2 was elevated in SND1-overexpressing cell culture supernatants (Fig. 3B). In addition, higher serum PGE2 levels were observed in osteosarcoma patients (12 cases) compared to healthy controls (23 cases) (Fig. 3C). Furthermore, the paired serum PGE2 levels were significantly positively correlated with the SND1 mRNA level in paired osteosarcoma tissues $(n=12)$ (Fig. 3D). Notably, the SND1 overexpression-stimulated cell proliferation was enhanced by exogenous addition of PGE2 (Fig. 3E and F). Collectively, these data revealed that SND1 promoted the proliferation of osteosarcoma cells by upregulating PGE2.
SND1 upregulates $C O X-2 / P G E 2$ expression through the $N F-\kappa B$ pathway. Since COX-2 levels have been revealed to be regulated by $\mathrm{NF}-\kappa \mathrm{B}$, the involvement of NF- $\mathrm{NB}$ in SND1-mediated PGE2 induction was investigated. To investigate $N F-\kappa B$ activity, SND1-overexpressing and control cells were transfected with a reporter vector $(3 \kappa \mathrm{B}-\mathrm{Luc})$ and treated with $\mathrm{TNF}-\alpha$ (a known activator of $N F-\kappa B$ ) and luciferase activity was then determined. As shown in Fig. 4A, the basal NF- $\kappa$ B activity was significantly higher in SND1-overexpressing cells compared to control cells. Furthermore, treatment with TNF- $\alpha$ markedly increased NF- $\kappa \mathrm{B}$ activity in SND1-overexpressing cells compared to Control cells (Fig. 4A). In SND1-overexpressing cells, the level of COX-2 protein expression was also significantly higher compared with that in control cells, and this phenomenon was further accentuated after treatment with TNF- $\alpha$ (Fig. 4B). In addition, siRNA was used to knockdown $\mathrm{NF}-\kappa \mathrm{B}$ p65 expression to determine if SND1 overexpression-stimulated COX-2/PGE2 expression depended on the $\mathrm{NF}-\kappa \mathrm{B}$ pathway. As revealed in Fig. $4 \mathrm{C}$ and $\mathrm{D}, \mathrm{NF}-\kappa \mathrm{B}$ p65 depletion by RNAi significantly inhibited the expression of COX-2 and PGE2 both in control and SND1-overexpressing cells, and this phenomenon was also further accentuated after treatment with TNF- $\alpha$. Collectively, these results indicated that SND1 activated the $\mathrm{NF}-\kappa \mathrm{B}$ pathway and increased COX-2/PGE2 expression.

SND1 promotes osteosarcoma tumor growth in vivo. To further explore the effect of SND1 on tumorigenesis in vivo, control or SND1-overexpressing cells were injected into nude mice. 
A
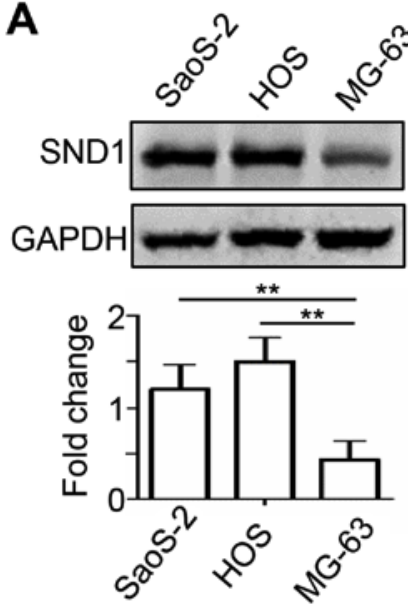

C

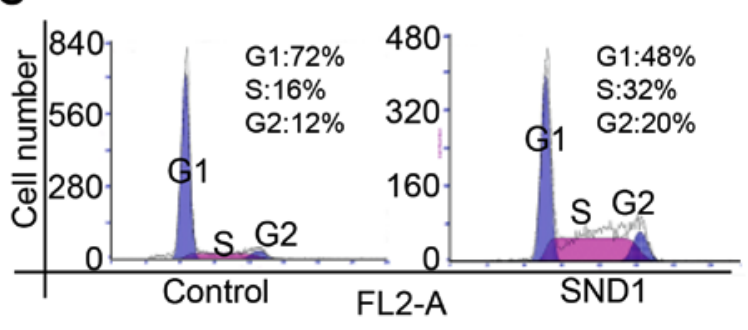

B
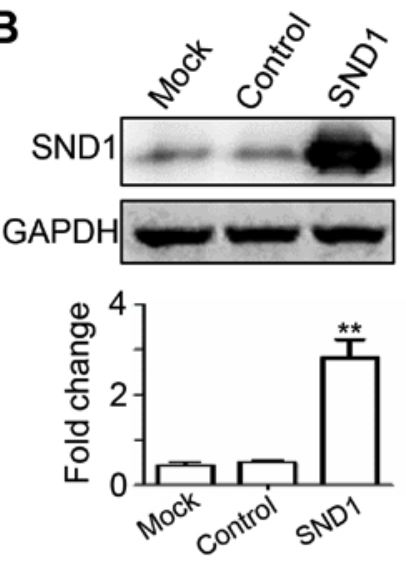

MG-63

D
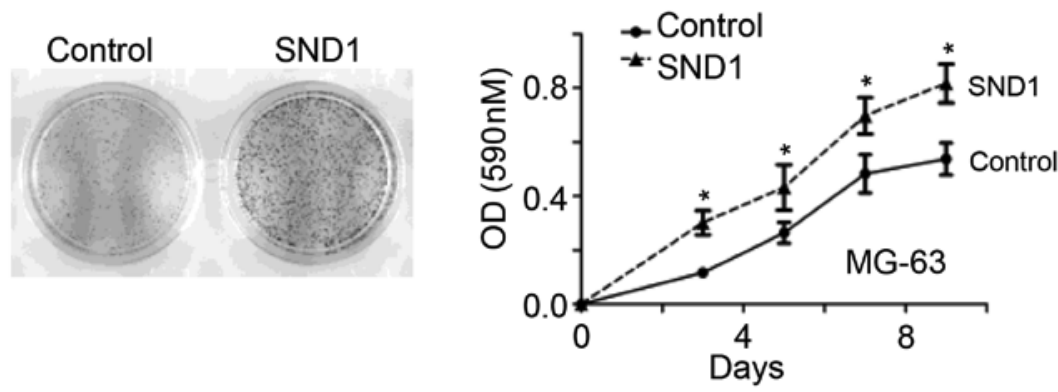

$\mathbf{E}$
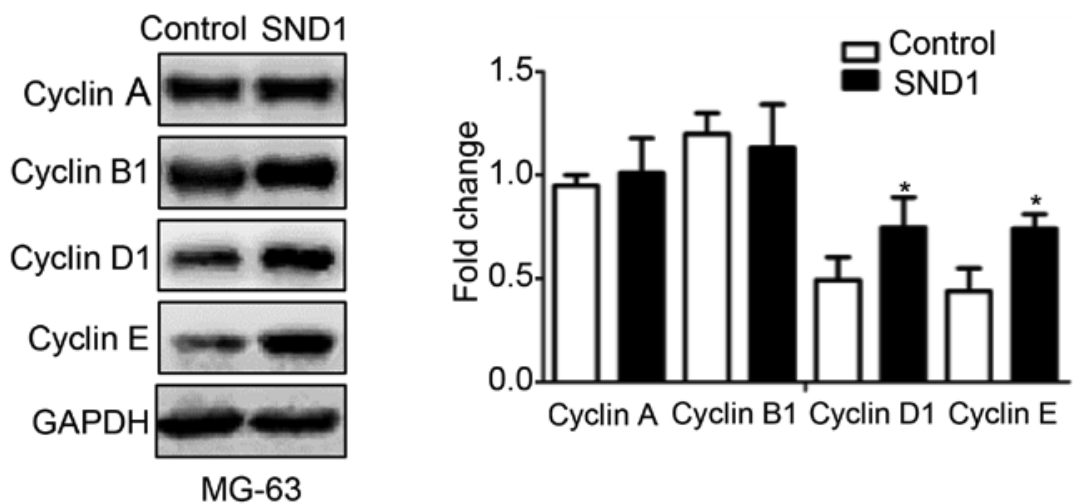

Figure 2. SND1 promotes the proliferation of osteosarcoma cells. (A) Western blot analysis of SND1 expression in 3 different osteosarcoma cell lines, namely MG-63, Saos-2 and HOS. Quantification of the protein levels is displayed in the bottom panel. (B) Western blot analysis of SND1 expression in mock, control (empty vector-transduced) or SND1-overexpressing (SND1 ORF-transduced) MG-63 cells. Quantification of the protein levels is displayed in the bottom panel. Statistical significance in A and B was determined by ANOVA-post-hoc-test analysis. (C) Flow cytometric analysis was performed on MG-63-control and MG-63-SND1-overexpressing cells. Quantification of cell populations is displayed in the right panel. (D) Cell proliferation assay was performed on MG-63-control and MG-63-SND1-overexpressing cells. Growth curves are displayed in the right panel. (E) Western blot analysis of cyclin protein expression in MG-63-control and MG-63-SND1-overexpressing cells. Quantification of the protein levels is displayed in the right panel. All of the relative protein expression was normalized by ImageJ (version no.: 1.8.0_112, https://imagej.nih.gov/ij/). Statistical significance in C, D and E was determined by a two-tailed, unpaired Student's t-test. All of the experiments were repeated three times. ${ }^{*} \mathrm{P}<0.05,{ }^{* * *} \mathrm{P}<0.01$. SND1, staphylococcal nuclease domain-containing 1.

In a 3-week assay, tumor volume and weight were monitored, and tumor growth was markedly enhanced with SND1 overexpression (Fig. 5A-C). The expression of COX-2 and PGE2 in SND1-overexpressing xenograft tissues was significantly higher than that of control groups, which was accompanied by increased PCNA levels (Fig. 5D and E). Collectively, these results revealed that SND1 promoted osteosarcoma tumor growth via COX-2/PGE2 production. 
A
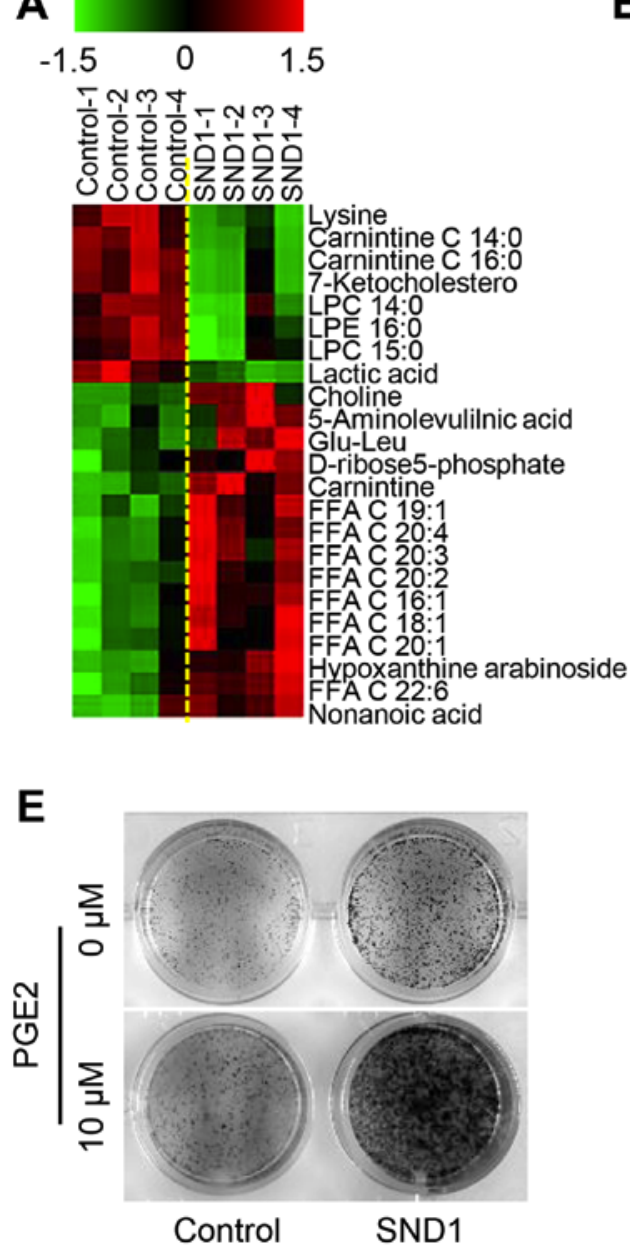

B
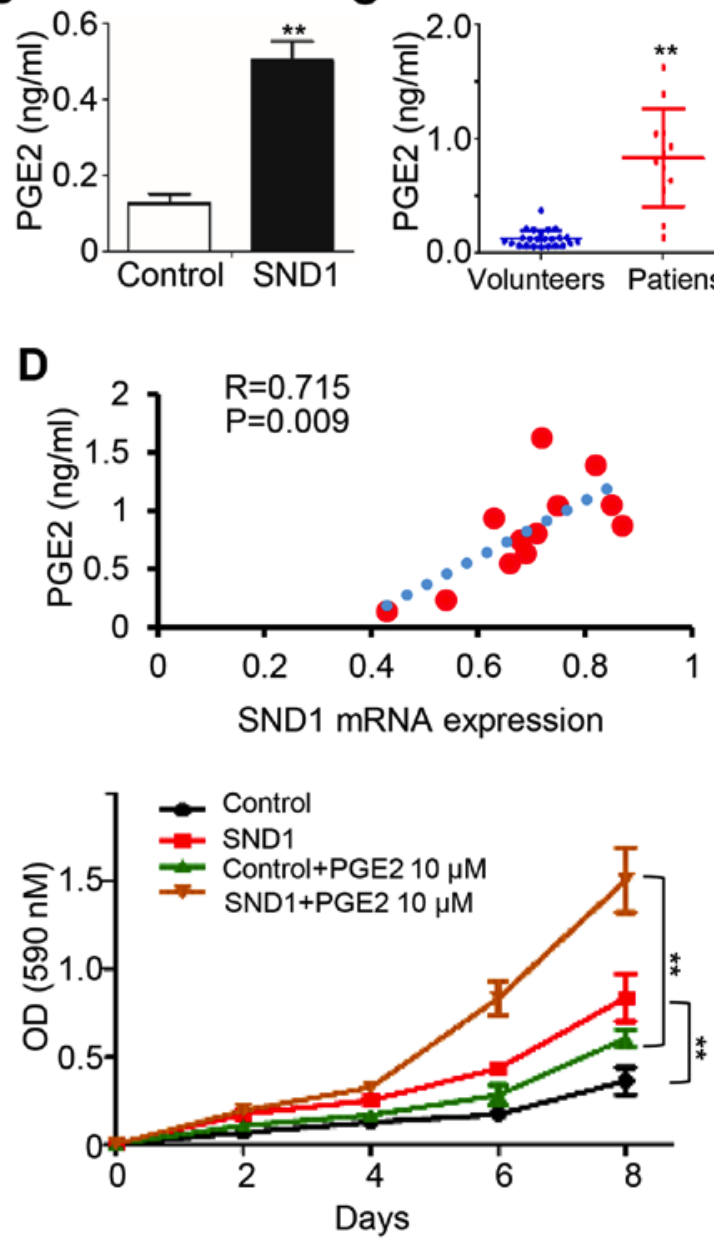

Figure 3. SND1 promotes PGE2 synthesis and release. (A) Heatmap of 23 differential metabolites between MG-63-Control and MG-63-SND1-overexpressing cells. Red indicates the increasing direction, and green indicates the decreasing direction. Statistical significance was evaluated by Mann-Whitney U test. (B) The PGE2 expression level in cell culture supernatants was assessed by ELISA. (C) The serum PGE2 expression level in osteosarcoma patients (12 cases) compared with healthy controls (23 cases) was assessed by ELISA. (D) Correlation analysis between SND1-mRNA in osteosarcoma fresh-frozen tissues and serum PGE2 level in paired blood samples. (E) Cell proliferation assay was performed on MG-63-control and MG-63-SND1-overexpressing cells with or without exogenous addition of PGE2. Growth curves are displayed in the right panel. Statistical significance in B, C and E was determined by the two-tailed, unpaired Student's t-test. The experiments were repeated three times. ${ }^{* *} \mathrm{P}<0.01$. SND1, staphylococcal nuclease domain-containing 1.

\section{Discussion}

Despite efforts that have been increasingly made to discover the underlying mechanisms of the malignant biological behavior of osteosarcoma, the specific mechanism is still not clear $(20,21)$. The present study revealed for the first time that overexpression of SND1 promoted osteosarcoma proliferation and tumorigenesis in vitro and in vivo. Moreover, the present findings demonstrated that SND1 manifested its pro-tumor activities by upregulating COX-2/PGE2 expression via the $\mathrm{NF}-\kappa \mathrm{B}$ pathway.

SND1 is a multifunctional protein that plays important roles in cell signal transduction, gene transcription, RNA splicing, RNA editing, stress, cell proliferation and apoptosis (11). Abnormally high expression of SND1 has been detected in a variety of tumors, such as colon $(22,23)$, lung $(24,25)$, prostate (26), breast (27) and liver cancer (28). However, the expression of SND1 in osteosarcoma is still unknown. The present study found that that mRNA and protein expression levels of SND1 were significantly higher in osteosarcoma tissue compared to paired normal bone tissues. In line with the present study, Bilbao-Aldaiturriaga et al reported that three SNPs located in miRNA-processing genes (CNOT1, CNOT4 and SND1) are associated with osteosarcoma susceptibility (29). Collectively, these results and studies provided an indication of the importance of SND1 in osteosarcoma.

Uncontrolled cell proliferation is an important feature of tumor cells. Recently, several studies have demonstrated that SND1 participates in cell cycle regulation, but the specific mechanism is still not clear. Yoo et al revealed that SND1 promoted the proliferation of human HCC cells by increasing RNA-induced silencing complex (RISC) activity (12). Jariwala et al confirmed that SND1 promoted the proliferation of human HCC cells by promoting tumor-initiating cell (TIC) formation (28). In addition, SND1 interacted with E2F-1, which upregulated E2F-1 transcriptional activity, thereby promoting cell proliferation (30). The present study demonstrated that SND1 significantly promoted MG-63 osteosarcoma cell proliferation and tumor growth in vivo and in vitro. 
A

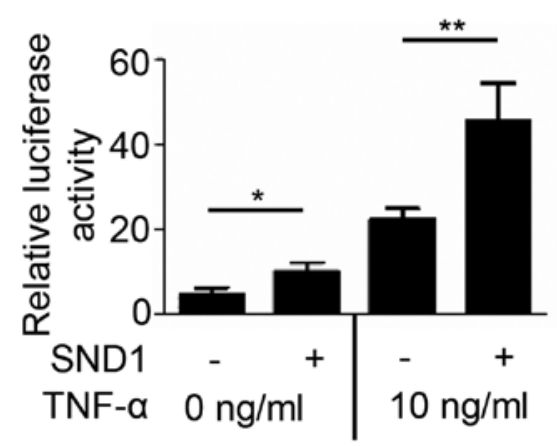

B
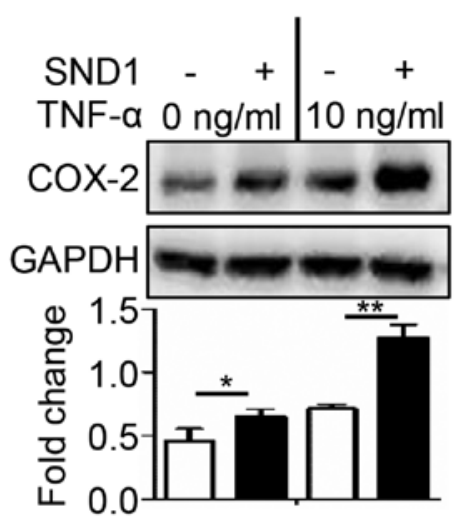
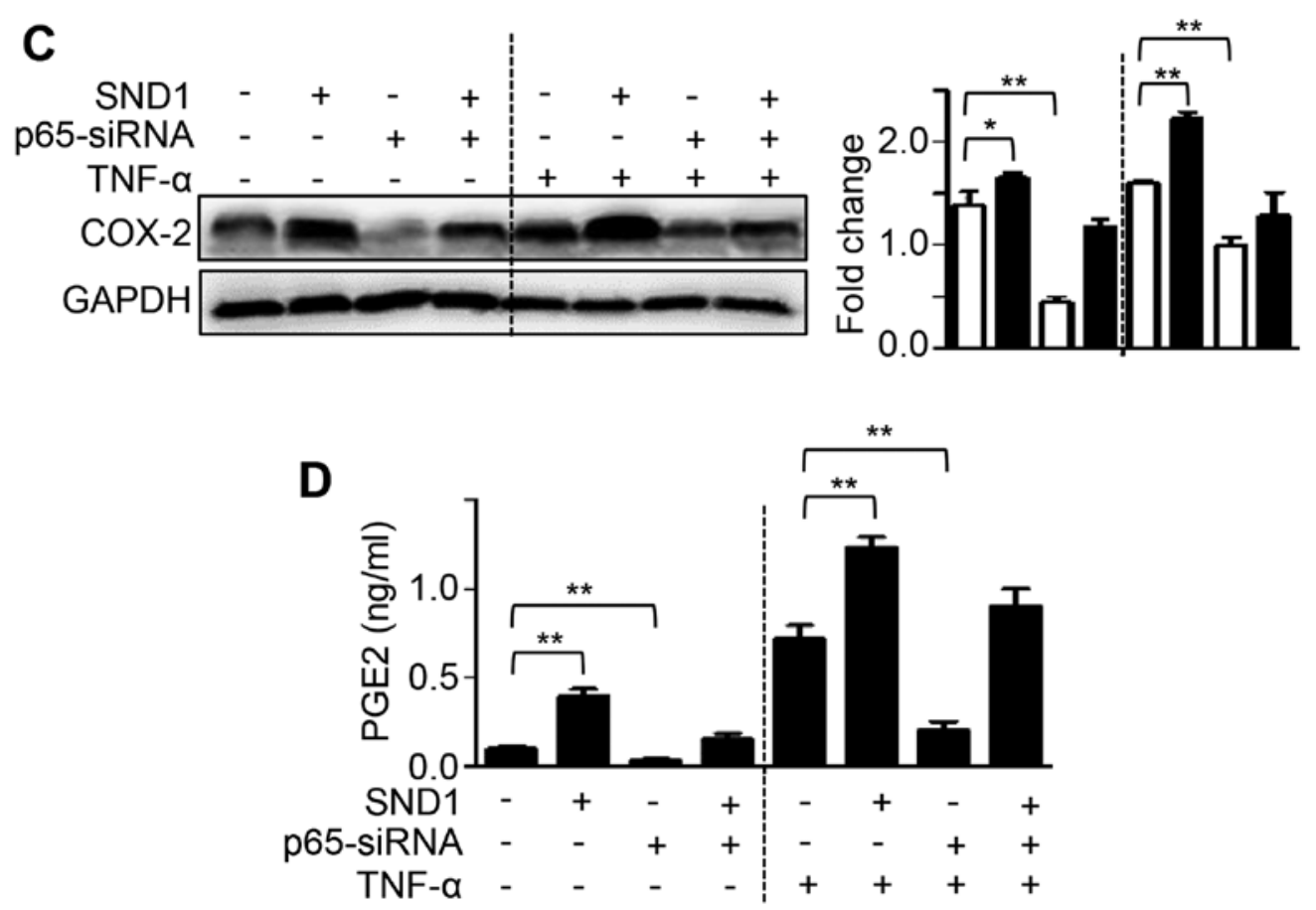

Figure 4. SND1 upregulates PGE2 expression via the NF- $\mathrm{B} / \mathrm{COX}-2$ pathway. (A) Luciferase assays of NF- $\mathrm{kB}$ activity in MG-63-control and MG-63-SND1-overexpressing cells, with or without TNF- $\alpha$ treatment $(10 \mathrm{ng} / \mathrm{ml})$ for $12 \mathrm{~h}$. (B) Western blot analysis of COX-2 expression in control and SND1-overexpressing cells with or without TNF- $\alpha(10 \mathrm{ng} / \mathrm{ml})$ treatment for $48 \mathrm{~h}$. Statistical significance in A and B was determined by a two-tailed, unpaired Student's t-test. (C) Western blot analysis of PGE2, SND1, COX-2 and p65 in different cells, with or without TNF- $\alpha$ treatment (10 ng/ml) for 48 h. (D) ELISA analysis of PGE2 expression level in different cell culture supernatants, with or without TNF- $\alpha$ treatment $(10 \mathrm{ng} / \mathrm{ml})$ for $48 \mathrm{~h}$. All of the relative protein expression was normalized by ImageJ (version no.: 1.8.0_112, https://imagej.nih.gov/ij/). Statistical significance in C and D was determined by ANOVA-post-hoc-test analysis. The experiments were repeated three times. ${ }^{*} \mathrm{P}<0.05,{ }^{* *} \mathrm{P}<0.01$. SND1, staphylococcal nuclease domain-containing 1.

In cancer cells, metabolism is markedly reprogrammed to support accelerated cell proliferation, including increased aerobic glycolysis and arachidonic acid/PG metabolism as well as decreased tricarboxylic acid cycle (31-33). The present study revealed that SND1 significantly increased the production of arachidonic acid and other $20 \mathrm{C}$ polyunsaturated fatty acids in MG-63 cells, which was accompanied by increased secretion of PGE2. A previous study reported that exogenous PGE2 treatment promoted the proliferation of osteosarcoma cells through the MAPK/ERK signaling pathway (34). In addition, PGE2 promoted the proliferation of colon cancer cells by activating the $\beta$-catenin axis through a biochemical pathway initiated by the activation of the G protein-linked PGE2 receptor, EP2 (35). The present study revealed that the expression level of serum PGE2 had a significant positive association with the SND1 expression level in osteosarcoma tissues. Collectively, these results indicated that SND1 may be involved in the proliferation of osteosarcoma cells by increasing the expression of PGE2.

COX-2 is the rate-limiting enzyme during the metabolic formation process of prostaglandins from arachidonic acid. Downregulation of COX-2 expression inhibits the proliferation of tumor cells and induces tumor cell apoptosis by reducing the synthesis of PG. In addition, high expression of COX-2 occurs in a variety of tumors, including osteosarcoma. The expression level of COX-2 is related to osteosarcoma staging, and it can be used as an independent prognostic factor in osteosarcoma (10). In recent years, the effect of selective COX-2 inhibitors on osteosarcoma has been confirmed in a number of trials (36). The present study revealed that SND1 promoted the proliferation of osteosarcoma cells via the upregulation of COX-2/PGE2 expression.

The present study also investigated the underlying mechanism of SND1-induced COX2/PGE2 upregulation. 
A

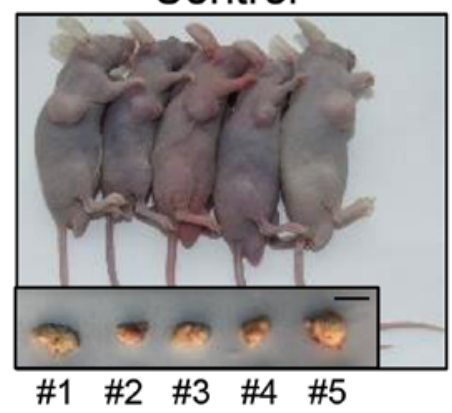

SND1

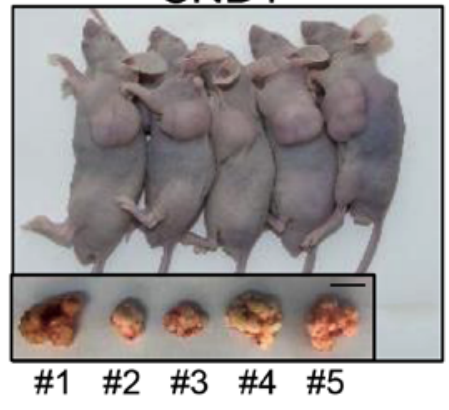

B

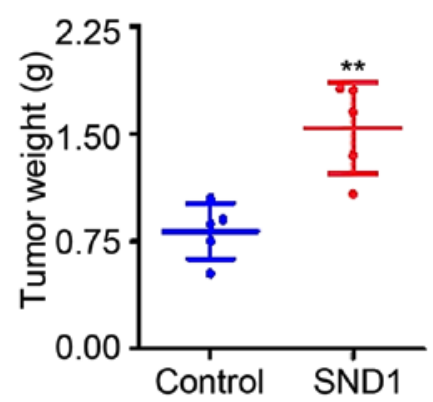

C
D

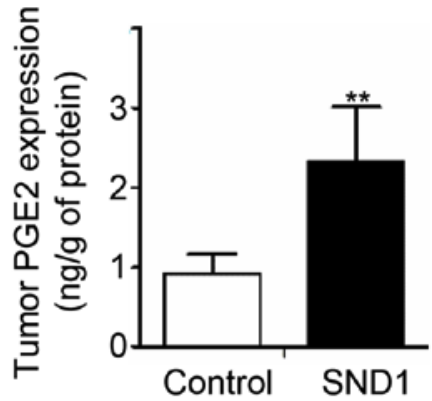

E


Figure 5. SND1 promotes osteosarcoma tumor growth in vivo. (A) Tumor images in mice subcutaneously injected with MG-63-control and MG-63-SND1-overexpressing-cells. Bar, $1 \mathrm{~cm}$. (B) Growth curves of xenografts-described in A. (C) Tumor weight of xenografts described in A. (D) PGE2 expression level in xenografts described in A. (E) Western blot analysis of SND1, COX-2 and PCNA expression in xenografts. Statistical significance was determined by a two-tailed, unpaired Student's t-test. ${ }^{*} \mathrm{P}<0.05,{ }^{* *} \mathrm{P}<0.01$. SND1, staphylococcal nuclease domain-containing 1.

$\mathrm{NF}-\kappa \mathrm{B}-\mathrm{p} 65$ is a subunit of the $\mathrm{NF}-\kappa \mathrm{B}$ transcription complex that plays a crucial role in inflammation, cell proliferation, differentiation, and survival. Several studies have revealed that the NF- $\mathrm{B}$ signaling pathway is in a continuous activation state in numerous tumors. PTGS2 is a target gene of p65, and activation of the NF- $\kappa \mathrm{B}$ pathway results in PTGS2 upregulation. Santhekadur et al revealed that SND1 increased NF- $\mathrm{BB}$ activation in HCC cells (16). The present study confirmed that SND1 also increased NF- $\kappa$ B activation in MG- 63 cells. In addition, depletion of p65 expression in SND1-ox cells abolished SND1 overexpression-stimulated PGE2 release and COX-2 protein expression. Collectively, these data revealed that SND1 promoted osteosarcoma cell proliferation and tumor growth via NF- $\kappa$ B-p65 activation. However, the mechanism by which SND1 activated NF- $\kappa$ B-p65 remains to be determined.

There are still some limitations in this study. First of all, the available osteosarcoma patients were very limited in our department, only 12 clinical samples were collected in this study. Due to this this small clinical volume, which is not enough to analyze the association between SND1 expression and clinical features, we simply counted the proportion of patients with higher SND1 expression under different clinical characteristics. In addition, no significant PGE2 expression change in the serum of the tumor-bearing mice was observed, and this may be associated with the reasons that follow: i) the model construction time was too short to reach the peak PGE2 concentration in the blood; ii) this was a subcutaneous rather than an orthotopic transplantation model.

In conclusion, these findings in osteosarcoma cells and xenografts support that SND1 promotes proliferation and tumor growth by upregulating COX-2/PGE2 expression via

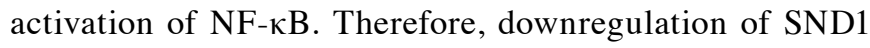
may represent an effective treatment method for inhibiting the proliferation of osteosarcoma. In addition, SND1 may act as a potential biomarker of the therapeutic strategies utilizing COX-2 inhibitors. 


\section{Acknowledgements}

Not applicable.

\section{Funding}

The present study was supported by the Science Research Project of Education of the Department of Liaoning Province (L2016022).

\section{Availability of data and materials}

The datasets used and/or analyzed during the current study are available from the corresponding author on reasonable request.

\section{Authors' contributions}

KS and JL conceived and designed the study. MZ, AW, BY, DW, SH and WZ performed the experiments. MZ and AW wrote the paper. All authors read and approved the manuscript and agree to be accountable for all aspects of the research in ensuring that the accuracy or integrity of any part of the work are appropriately investigated and resolved.

\section{Ethics approval and consent to participate}

Ethical approval for the project was obtained from the Research Ethics Committee of the First Affiliated Hospital of Dalian Medical University. All patients signed the informed consent. The non-retrospective ethical approval obtained for the animal experiments was obtained from Dalian Medical University Research Ethics Committee.

\section{Patient consent for publication}

Not applicable.

\section{Competing interests}

The authors declare that they have no competing interests.

\section{References}

1. Hingorani P, Janeway K, Crompton BD, Kadoch C, Mackall CL, Khan J, Shern JF, Schiffman J, Mirabello L, Savage SA, et al: Current state of pediatric sarcoma biology and opportunities for future discovery: A report from the sarcoma translational research workshop. Cancer Genet 209: 182-194, 2016.

2. Harrison DJ, Geller DS, Gill JD, Lewis VO and Gorlick R: Current and future therapeutic approaches for osteosarcoma. Expert Rev Anticancer Ther 18: 39-50, 2018.

3. Smith WL, DeWitt DL and Garavito RM: Cyclooxygenases: Structural, cellular, and molecular biology. Annu Rev Biochem 69: 145-182, 2000.

4. Pang LY, Hurst EA and Argyle DJ: Cyclooxygenase-2: A role in cancer stem cell survival and repopulation of cancer cells during therapy. Stem Cells Int 2016: 2048731, 2016.

5. Xu L, Stevens J, Hilton MB, Seaman S, Conrads TP, Veenstra TD Logsdon D, Morris H, Swing DA, Patel NL, et al: COX-2 inhibition potentiates antiangiogenic cancer therapy and prevents metastasis in preclinical models. Sci Transl Med 6: 242ra84, 2014.

6. Rodriguez PC, Hernandez CP, Quiceno D, Dubinett SM, Zabaleta J, Ochoa JB, Gilbert J and Ochoa AC: Arginase I in myeloid suppressor cells is induced by COX-2 in lung carcinoma. J Exp Med 202: 931-939, 2005.
7. Cha YI and DuBois RN: NSAIDs and cancer prevention: Targets downstream of COX-2. Annu Rev Med 58: 239-252, 2007.

8. Naruse T, Nishida Y, Hosono K and Ishiguro N: Meloxicam inhibits osteosarcoma growth, invasiveness and metastasis by COX-2-dependent and independent routes. Carcinogenesis 27 : 584-592, 2006

9. Qu L and Liu B: Cyclooxygeanse-2 promotes metastasis in osteosarcoma. Cancer Cell Int 15: 69, 2015.

10. Rodriguez NI, Hoots WK, Koshkina NV, Morales-Arias JA, Arndt CA, Inwards CY, Hawkins DS, Munsell MF and Kleinerman ES: COX-2 expression correlates with survival in patients with osteosarcoma lung metastases. J Pediatr Hematol Oncol 30: 507-512, 2008.

11. Gutierrez-Beltran E, Denisenko TV, Zhivotovsky B and Bozhkov PV: Tudor staphylococcal nuclease: Biochemistry and functions. Cell Death Differ 23: 1739-1748, 2016.

12. Yoo BK, Santhekadur PK, Gredler R, Chen D, Emdad L, Bhutia S, Pannell L, Fisher PB and Sarkar D: Increased RNA-induced silencing complex (RISC) activity contributes to hepatocellular carcinoma. Hepatology 53: 1538-1548, 2011.

13. Jariwala N, Rajasekaran D, Srivastava J, Gredler R, Akiel MA, Robertson CL, Emdad L, Fisher PB and Sarkar D: Role of the staphylococcal nuclease and tudor domain containing 1 in oncogenesis (review). Int J Oncol 46: 465-473, 2015.

14. Pandey MK, Gupta SC, Nabavizadeh A and Aggarwal BB: Regulation of cell signaling pathways by dietary agents for cancer prevention and treatment. Semin Cancer Biol 46: 158-181, 2017.

15. Tang QL, Xie XB, Wang J, Chen Q, Han AJ, Zou CY, Yin JQ, Liu DW, Liang Y, Zhao ZQ, et al: Glycogen synthase kinase-3 $\beta$, NF- $\kappa \mathrm{B}$ signaling, and tumorigenesis of human osteosarcoma. J Natl Cancer Inst 104: 749-763, 2012.

16. Santhekadur PK, Das SK, Gredler R, Chen D, Srivastava J, Robertson C, Baldwin AS Jr, Fisher PB and Sarkar D: Multifunction protein staphylococcal nuclease domain containing 1 (SND1) promotes tumor angiogenesis in human hepatocellular carcinoma through novel pathway that involves nuclear factor $\mathrm{\kappa B}$ and miR-221. J Biol Chem 287: 13952-13958, 2012.

17. Livak KJ and Schmittgen TD: Analysis of relative gene expression data using real-time quantitative PCR and the $2^{-\Delta \Delta C \mathrm{~T}}$ method. Methods 25: 402-408, 2001.

18. Piao H, Yuan Y, Wang M, Sun Y, Liang H and Ma L: $\alpha$-catenin acts as a tumor suppressor in E-cadherin-negative basal-like breast cancer by inhibiting NF- $\mathrm{BB}$ signaling. Nat Cell Biol 16: 245-254, 2014.

19. Wu Q, Zhou L, Sun X, Yan Z, Hu C, Wu J, Xu L, Li X, Liu H, Yin P, et al: Altered lipid metabolism in recovered SARS patients twelve years after infection. Sci Rep 7: 9110, 2017.

20. Lin YH, Jewell BE, Gingold J, Lu L, Zhao R, Wang LL and Lee DF: Osteosarcoma: Molecular pathogenesis and iPSC modeling. Trends Mol Med 23: 737-755, 2017.

21. Taran SJ, Taran R and Malipatil NB: Pediatric osteosarcoma: An updated review. Indian J Med Paediatr Oncol 38: 33-43, 2017.

22. Tsuchiya $N$, Ochiai M, Nakashima K, Ubagai T, Sugimura T and Nakagama H: SND1, a component of RNA-induced silencing complex, is up-regulated in human colon cancers and implicated in early stage colon carcinogenesis. Cancer Res 67: 9568-9576, 2007.

23. Wang N, Du X, Zang L, Song N, Yang T, Dong R, Wu T, He X and Lu J: Prognostic impact of Metadherin-SND1 interaction in colon cancer. Mol Biol Rep 39: 10497-10504, 2012.

24. Xing A, Pan L and Gao J: p100 functions as a metastasis activator and is targeted by tumor suppressing microRNA-320a in lung cancer. Thorac Cancer 9: 152-158, 2018

25. Jin SJ, Lee A, Li J, Liyanage H, Yang Y, Guo L, Asmann YW, Li PW, Erickson-Johnson M, Sakai Y, et al: Common oncogene mutations and novel SNDI-BRAF transcript fusion in lung adenocarcinoma from never smokers. Sci Rep 5: 9755, 2015.

26. Kuruma H, Kamata Y, Takahashi H, Igarashi K, Kimura T, Miki K, Miki J, Sasaki H, Hayashi N and Egawa S: Staphylococcal nuclease domain-containing protein 1 as a potential tissue marker for prostate cancer. Am J Pathol 174: 2044-2050, 2009.

27. Ho J, Kong JW, Choong LY, Loh MC, Toy W, Chong PK, Wong $\mathrm{CH}$, Wong CY, Shah N and Lim YP: Novel breast cancer metastasis-associated proteins. J Proteome Res 8: 583-594, 2009.

28. Jariwala N, Rajasekaran D, Mendoza RG, Shen XN, Siddiq A, Akiel MA, Robertson CL, Subler MA, Windle JJ, Fisher PB, et al: Oncogenic role of SND1 in development and progression of hepatocellular carcinoma. Cancer Res 77: 3306-3316, 2017. 
29. Bilbao-Aldaiturriaga N, Gutierrez-Camino A, Martin-Guerrero I, Pombar-Gomez M, Zalacain-Diez M, Patiño-Garcia A, Lopez-Lopez E and Garcia-Orad A: Polymorphisms in miRNA processing genes and their role in osteosarcoma risk. Pediatr Blood Cancer 62: 766-769, 2015.

30. Su C, Zhang C, Tecle A, Fu X, He J, Song J, Zhang W, Sun X, Ren Y, Silvennoinen O, et al: Tudor staphylococcal nuclease (Tudor-SN), a novel regulator facilitating $\mathrm{G}_{1} / \mathrm{S}$ phase transition, acting as a co-activator of E2F-1 in cell cycle regulation. J Biol Chem 290: 7208-7220, 2015.

31. Netea-Maier RT, Smit Jwa and Netea MG: Metabolic changes in tumor cells and tumor-associated macrophages: A mutual relationship. Cancer Lett 413: 102-109, 2018.

32. Yarla NS, Bishayee A, Sethi G, Reddanna P, Kalle AM, Dhananjaya BL, Dowluru KS, Chintala R and Duddukuri GR: Targeting arachidonic acid pathway by natural products for cancer prevention and therapy. Semin Cancer Biol 40-41: 48-81, 2016.
33. Zhou Z, Ibekwe E and Chornenkyy Y: Metabolic alterations in cancer cells and the emerging role of oncometabolites as drivers of neoplastic change. Antioxidants 7: E16, 2018.

34. Krysan K, Reckamp KL, Dalwadi H, Sharma S, Rozengurt E, Dohadwala $M$ and Dubinett SM: Prostaglandin $\mathrm{E}_{2}$ activates mitogen-activated protein kinase/Erk pathway signaling and cell proliferation in non-small cell lung cancer cells in an epidermal growth factor receptor-independent manner. Cancer Res 65: 6275-6281, 2005.

35. Castellone MD, Teramoto H, Williams BO, Druey KM and Gutkind JS: Prostaglandin $\mathrm{E}_{2}$ promotes colon cancer cell growth through a $\mathrm{G}_{\mathrm{s}}$-axin- $\beta$-catenin signaling axis. Science 310 : 1504-1510, 2005 .

36. Consalvi S, Biava M and Poce G: COX inhibitors: A patent review (2011-2014). Expert Opin Ther Pat 25: 1357-1371, 2015. 